\title{
Decoupling Vibration and Electron Energy Dependencies in the Photoelectron Circular Dichroism of a Terpene, 3-carene
}

\author{
Hassan Ganjitabar, ${ }^{[a]}$ Gustavo Garcia, ${ }^{[b]}$ Laurent Nahon, $^{[b]}$ and Ivan Powis*[a] \\ ${ }^{[a]}$ School of Chemistry, The University of Nottingham, University Park, Nottingham NG7 2RD, UK \\ ${ }^{[b]}$ Synchrotron SOLEIL, I'Orme des Merisiers, Saint Aubin BP 48, 91192 Gif sur Yvette Cedex, France. \\ *Corresponding Author:
}

Email: ivan.powis@nottingham.ac.uk

Phone: +44 1159513467

Keywords: Photoelectron Circular Dichroism • PECD • Vibrational Dynamics • Electron Kinetic Energy

- Vibrational Spectroscopy 


\section{Abstract:}

A fresh perspective on the interaction of electron and nuclear motion in photon induced dynamical processes can be provided by the coupling of photoelectron angular distributions and cation vibrational states in the photoionization of chiral molecules using circularly polarized radiation. The chiral contributions, manifesting as a forward-backward asymmetry in the photoemission, can be assessed using Photoelectron Circular Dichroism (PECD) which has revealed enhanced vibrational influence exerted on the outgoing photoelectron. In this paper, we investigate PECD of a rigid chiral monoterpene, 3-carene, using single-photon VUV ionization by polarized synchrotron radiation, and selecting energies from ionization threshold up to $19.0 \mathrm{eV}$. By judicious choice of these photon energies, two factors that influence PECD asymmetry values, electron kinetic energy and ion vibrational level, can be effectively isolated, allowing a clear demonstration of the very marked vibrational effects. A slow photoelectron spectrum (SPES) is used to examine the vibrational structure of the isolated outermost valence (HOMO) photoelectron band and peak assignments are made with the aid of a Franck-Condon simulation. Together, these provide an estimate of the adiabatic ionization energy as $8.385 \mathrm{eV}$. The reported chiral asymmetry from the randomly oriented 3-carene enantiomers reaches a maximum of over $21 \%$. Theoretical PECD calculations, made both for the fixed equilibrium molecular geometry and also modelling selected normal mode vibration effects, are presented to provide further insight.

\section{Introduction}

Photoionization of randomly-oriented enantiomers of a chiral molecule by circularly polarized light $(C P L)$ opens up a rich source of information on the dynamics of electrons in the field of the molecular nuclei when the laboratory frame photoelectron angular emission is recorded. These angular distributions contain additional antisymmetric contributions, that either are necessarily absent in achiral target molecule-photon interactions, or will average to zero with racemic mixtures or nonelliptical polarization states. ${ }^{1,2}$ Crucially, the additional chiral term(s) in the angular distribution introduce a greater sensitivity to the phase-shifts induced by electron scattering off the molecular potential as compared to the more familiar symmetric contributions (typified by the established anisotropy parameter, $\beta$ ). The chiral contribution is seen as a forward-backward asymmetry (referenced to the direction of a CPL ionizing photon beam) in the photoelectron angular emission, and this reverses with exchange of either the enantiomer or the CPL handedness. Photoelectron Circular Dichroism (PECD) records the angular distribution using alternatively left- and right- CPL radiation and thence provides quantitative measures of the chiral asymmetry. ${ }^{2-5}$ 
The photoelectron energy dependence of PECD provides a clear spectral signature distinguishing the initial orbital ionized (even when these may be poorly resolved in the photoelectron spectrum ${ }^{6,7}$ ) but perhaps more importantly responds to structural features such as isomerism, 8,9 conformation, 5,6,10-14 chemical substitution, ${ }^{15,16}$ dimerization and clustering, ${ }^{17-19}$ and above all absolute configuration. ${ }^{13}$

While the foregoing lists essentially static structural sensitivities, a very significant finding was the observation that the excitation of a weak vibrational mode in the cation of methyloxirane caused the PECD asymmetry to completely reverse direction. ${ }^{20}$ Subsequently, this was proposed to be a consequence of a partial phase-shift cancellation in the photoionization matrix elements that could occur under certain conditions when odd vibrational quanta were excited. ${ }^{21}$ This suggestion has been more quantitatively evaluated to characterize the different degrees of vibrational sensitivity observed in tri-fluoromethyloxirane. ${ }^{22}$ Nevertheless, a complete validation is required.

In the interim, further strong modulations of the PECD asymmetry with vibrational structure of the corresponding molecular photoelectron spectrum (PES) has been noted in several monoterpene $\left(\mathrm{C}_{10} \mathrm{H}_{16}\right)$ isomers, ${ }^{8}$ and has been most extensively studied for one of these terpenes, limonene. ${ }^{23}$ In this molecule strong fluctuations of the PECD signal over a wide photon energy range were recorded, with peaks that correlate with the major vibrational peaks in the PES HOMO band. In the valleys between these principal peaks the PECD can again be observed to sometimes reverse sign (photoelectron emission direction). But while providing an unambiguous demonstration of vibrational coupling effects, a full interpretation was limited by two factors. First, the high energy side of the limonene HOMO PES band strongly overlaps the adjacent HOMO-1 orbital's ionization, with the relative contributions in the overlap region undefined. Secondly, limonene possesses six different conformers, with estimates suggesting that three may be populated at room temperature. Even under cooled experimental conditions, the population can at best only be inferred from calculated energetics and estimated sample temperatures.

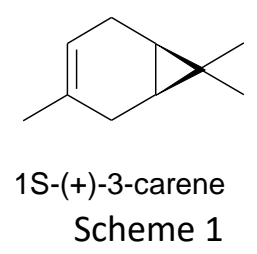

In this paper we study another of these terpene isomers, 3-carene. It possesses a bicyclic ring structure comprised of six-membered and three-membered rings. The HOMO is a localised $\pi$ orbital at the $C=C$ double bond. For a relatively large molecule the first HOMO band of the carene PES displays very well differentiated vibrational peaks ${ }^{8}$ and, as an object for the study of vibrationally resolved PECD, it eliminates the two above mentioned limitations of limonene, namely : (a) overlapping of the HOMO 
and HOMO-1 PES bands is minimized; and (b) the rigid bicyclic structure means that there are no longer multiple conformers to be considered. In fact theoretical calculations ${ }^{24}$ (CCSD/aug-cc-pVDZ, B3LYP/aug-cc-pVTZ) identify a single stable conformer with a near planar six-membered ring.

Photoionization dipole matrix elements are functions of the continuum electron momentum, and consequently the resulting PECD asymmetries to which they give rise are electron energy dependent, sometimes strongly so. As an extreme example of this, PECD of another $\mathrm{C}_{10} \mathrm{H}_{16}$ isomer, $\alpha$-pinene, records asymmetry factors that range monotonically from nearly zero up to $40 \%$ as the electron energy changes by $1.5 \mathrm{eV} .{ }^{8}$ Perhaps more typically, vibrationally unresolved PECD asymmetries have been reported that vary continuously, including possible changes in sign, across the energy ranges being studied. For ionization of any particular orbital, with a given ionization energy, there will be an increase in emitted electron kinetic energy (eKE) with increasing photon energy. Conversely, for a given orbital and photon energy the eKE would be reduced by any increase in vibrational excitation energy. In principle, then, variations in the PECD associated with passage across the eKE axis of a photoelectron spectrum may be attributable both to the simple direct increase in electron energy or to more subtle changes in the dipole matrix elements influenced by a varying vibrational level population. While the observation of a strong correlation between the positions of peak structure in the PES and PECD can give a solid indication of some vibration-electron coupling, for a more complete understanding of this clear breaking of the FC approximation, it is essential to attempt to disentangle these contributing effects.

That is a goal for the present work. By a judicious selection of photon energies we will examine PECD at successive vibrational levels, but always maintaining the same electron energy; alternatively we will focus on a selected vibrational peak, and examine the variation in PECD for different electron kinetic energies. The vibrational structure observed in the 3-carene PES will be assigned by using a FranckCondon (FC) simulation for comparison with the PES. CMS-X $\alpha$ calculations made at the fixed neutral equilibrium geometry of 3-carene will be used to predict and map-out the expected simple eKE dependence of the HOMO ionization without consideration of vibrational effects. While full vibrational PECD simulations are not currently feasible for systems of this size (with 72 normal modes) extended calculations are reported that consider excitation of a few selected vibrational modes in order to provide a more qualitative insight into the potential influence of electron-nuclei coupling on PECD asymmetries.

\section{Experimental Details}

Measurements were made at the DESIRS vacuum ultraviolet (VUV) beamline ${ }^{25}$ at the SOLEIL synchrotron (France), using the DELICIOUS-3 double imaging (i ${ }^{2}$ PEPICO) spectrometer ${ }^{26}$ installed at the 
permanent molecular beam end-station SAPHIRS. ${ }^{27}$ Fully variable polarized light was provided by an electromagnetic planar/helicoidal undulator, HU640, offering pure circular polarisation $\left(\left|S_{3}\right|>0.97\right)$ over the whole energy range to be used. For photon energies below $15 \mathrm{eV}$ an in-line gas filter upstream of the 6.65 meter long normal incidence monochromator was filled with 0.24 mbar of Ar to remove undulator harmonics. ${ }^{28}$

The DELICIOUS-3 spectrometer couples a modified Velocity Map Imaging (VMI) spectrometer for angularly resolved electron detection with an ion imaging/modified Wiley McLaren time of flight analyser for ion detection. Detecting the electrons and the ions in coincidence mode allows photoelectron VMI images (hence also the PES and PECD) to be ion-mass filtered. All the results reported below are obtained by analysing the photoelectrons in coincidence with the parent mass ( $\mathrm{m} / \mathrm{z}=136 \mathrm{amu}$ ) which can help suppress both unwanted background ionization and any scattered electrons.

Adopting a previously detailed protocol, ${ }^{7}$ PECD measurements were made at selected photon energies by alternating Left (LCP) and Right (RCP) Circular Polarisation every 15 minutes. After a number of such cycles all the VMI images recorded with a given polarization were added to form a single pair of LCP \& RCP images. The VMI-PES was extracted from the total image $L C P+R C P$ while the PECD spectra were obtained from difference image LCP-RCP, using the pBaseX algorithm. ${ }^{29}$ In the case of single-photon ionization and randomly-oriented targets, the anticipated photoelectron angular distributions can be expressed as

$$
I^{\{p\}}(\theta)=1+b_{1}^{\{p\}} P_{1}(\cos \theta)+b_{2}^{\{p\}} P_{2}(\cos \theta)
$$

where $P_{i}$ are Legendre polynomials, $p$ is an helicity index indicating the light polarisation (with $p=0$ for the linear polarisation and $p= \pm 1$ for left/right CP), and $\theta$ is the emission direction (referred to the photon beam propagation direction in the case of $\mathrm{CP}$ ). Due to symmetry properties of the angular parameters, $b_{i}^{p}$, the normalised dichroism LCP-RCP simplifies to provide

$$
\operatorname{PECD}=I^{\{+1\}}(\theta)-I^{\{-1\}}(\theta)=2 b_{1}^{\{+1\}} P_{1}(\cos \theta) .
$$

A more wide-ranging photoelectron spectrum of 3-carene was recorded by scanning the photon energy in $5 \mathrm{meV}$ steps with a fixed horizontal polarization. Threshold and low energy electrons were recorded at each scan point using the DELICIOUS-3 electron VMI. The signal on the resulting 2D matrix of electron counts (photon energy vs. eKE) is integrated over the constant ionic state lines. Then by setting an upper limit on the eKE integration window an acceptable compromise between resolution and $\mathrm{S} / \mathrm{N}$ was achieved. Further details of the SPES method can be found elsewhere. ${ }^{30,31}$ 
In all cases S-(+)-3-carene was introduced to the spectrometer by flowing He carrier gas (0.5 bar pressure) through a bubbler containing the liquid sample at room temperature. The He/vapour mix was then expanded into vacuum through a $70 \mu \mathrm{m}$ diameter nozzle. After passage through a doubleskimmer arrangement, the seeded molecular beam entered the main spectrometer chamber. Enantiomeric samples of S-(+)-3-carene were obtained from Sigma-Aldrich and the supplied enantiomeric excess was verified to be $\geq 99 \%$ by a two-column gas chromatography (GC2) chiral analysis.

\section{Computational Details}

Geometry optimisation and harmonic frequency calculation for both the neutral and cationic ground states of 3-carene were done using Gaussian 16 software ${ }^{32}$ with B3LYP/cc-pVTZ and MP2/6-31G** model chemistries. These normal mode analyses were then used to perform a Franck-Condon $0 \mathrm{~K}$ simulation of the HOMO band PES using Gaussian 16 and FC-Lab II ${ }^{33}$ software. For these simulations harmonic frequencies were scaled by either 0.95 (MP2) or 0.98 (B3LYP). Vertical ionization energies were calculated at the B3LYP optimized equilibrium geometry using the OVGF method with aug-ccpVDZ and cc-pVTZ basis sets.

Electric dipole matrix elements for the photoionization process were calculated using the continuum multiple scattering method (CMS-X $\alpha$ ) with a Slater $X \alpha$ exchange potential ${ }^{34,35}$ following procedures as previously described. ${ }^{11,36-38}$ In this study the X $\alpha$ model potential was constructed using overlapping spherical regions placed at the B3LYP/cc-pVTZ optimized atomic centres and with a radius calculated by the Norman algorithm ${ }^{39}$ then scaled by a factor 0.81 . A spherical harmonic angular basis truncated at $\mathrm{l}_{\max }=18,4,3$ (referring, respectively to the asymptotic region, the $\mathrm{C}$ atomic regions, and the $\mathrm{H}$ atomic regions) was employed for the continuum electron calculations while for the initial neutral state a smaller basis $\left(l_{\max }=6,2,1\right)$ sufficed. Subsequently, chiral asymmetry parameters, $b_{1}$, and PECD were calculated using the photoionization dipole matrix elements derived from this potential model.

The fixed geometry CMS-X $\alpha$ calculations were extended to simulate the likely vibrational influence of selected vibrational modes. For this purpose, the complex dipole matrix elements were averaged over the normal coordinate of the chosen mode, weighted by the harmonic vibrational wavefunction overlap. All other modes were held frozen at their equilibrium position during these calculations. ${ }^{21}$

\section{Results and Discussion}

The total and mass-selected SPES of 3-carene recorded in coincidence with the indicated parent and fragment ion masses and spanning the ionization energy range $8.0 \mathrm{eV}-10.5 \mathrm{eV}$, are shown in Figure 
1. It can be seen that the onset of the first fragmentation channel $(\mathrm{m} / \mathrm{z}$ 93) lies above $9.05 \mathrm{eV}$ as previously determined ${ }^{40}$ and the majority of the first HOMO band region is undissociated. It is also apparent that above $\sim 9 \mathrm{eV}$, on the high energy edge of the HOMO band there is most likely some limited overlap with the adjacent HOMO-1 ionization; nevertheless this is much more limited than in limonene and overall creates a much lesser complication for the interpretation. ${ }^{23}$ Furthermore, filtering the VMI images by selecting electrons coincident with the parent ion mass ( $\mathrm{m} / \mathrm{z} 136)$ above this strongly discriminates against electrons emitted in the dissociative HOMO-1 ionization with no detrimental effects below $h v=9 \mathrm{eV}$. It also helps eliminate scattered electrons and, above $h v=12.6$ $\mathrm{eV}$, any ionization of residual $\mathrm{H}_{2} \mathrm{O}$. Subsequent VMI data and results will therefore employ the parent mass channel filtering.

VMI-PES and VMI-PECD measurements were made at photon energies ranging from $8.55 \mathrm{eV}$, just above the ionization threshold, up to $19 \mathrm{eV}$. Figure 2 shows two examples of the VMI PES and associated PECD recorded at $h v=10.5 \mathrm{eV}$ and $h v=12 \mathrm{eV}$. It is apparent that as the energies increase the VMI-PES absolute resolution degrades, since the $\mathrm{VMI}$ offers a constant resolving power, $\triangle E / E$. But while the PES vibrational structure starts to smear out, a clear modulation of the accompanying PECD values that correlates with the known position of vibrational peaks persists. The PECD measurements are relatively similar (with small offset) in the region of the first peaks, $A$ and $B$ (as identified in Fig. 1), but the trends show more divergence around $C$ and $D$. In region $D$ and above, the PECD at both photon energies displays more structure than the corresponding VMI-PES extracted from the same images, a phenomenon we have noted previously. ${ }^{6,7}$ The VMI PES and PECD results are, however, filtered on parent mass to discriminate against the dissociative (HOMO-1) ${ }^{-1}$ state, but it cannot be completely discounted that some additional structure in the high energy PECD may be attributable to this underlying HOMO-1 ionization.

\section{A: PECD at the origin}

Before presenting a detailed vibrational analysis of the HOMO ionization we can consider an overview of the energy dependent PECD with minimal reference to the detail of any accompanying vibrational excitation. Taking the ionization energy of the first PES peak $A$ as an approximate indication of the vibrationless origin (vide infra) one can record the accompanying PECD values in this region ( $~ 8.4 \mathrm{eV}$ ) and consider them to represent the PECD in the absence of vibrational excitation. These values are

then expressed as $b_{1}^{\{+1\}}(=\mathrm{PECD} / 2)$ and plotted as a function of electron kinetic energy in Figure 3. Also included is the result of the CMS-Xa calculation made at the fixed neutral molecule equilibrium geometry. There is excellent theory-experiment agreement displayed by these results, maintained to within $1 \mathrm{eV}$ of threshold, where electron correlation (absent in the $\mathrm{X} \alpha$ potential) might be expected 
to become more important. It is also notable that between $1 \mathrm{eV}$ and $2 \mathrm{eV}$ kinetic energy, the PECD asymmetry factor leaps from around $-20 \%$ to approximately zero over a range of just one $\mathrm{eV}$.

\section{B: PECD-Vibration Resolved PES Correlation}

We now turn to a more detailed examination of the PECD measured across the vibrational profile of the HOMO PES band. In Figure 4 PECD recorded at photon energies up to $9.5 \mathrm{eV}$, the region offering best VMI photoelectron resolution, is plotted out, and can be compared with arbitrarily scaled example of the VMI-PES and SPES plotted on the same energy axis. As already noted, there is a strong correlation between the PES and PECD peaks. It is also evident from Fig. 4 that there is an additional dispersion in the range of PECD values with varying photon energy, similar to that observed for the related monoterpene, limonene. ${ }^{23}$ However, whereas in limonene the displacement of entire PECD curves at successively increased photon energies occurs monotonically, here the pattern is more involved. This can be attributed to the rapid variation of PECD with electron energy revealed in Fig. 3. It is therefore desirable to attempt to separate out electron energy and cation vibrational effects in order to obtain a clearer picture of the latter.

To better isolate vibrational influences, VMI-PES and PECD data recorded at photon energies $h v=8.7$, $8.85,9.0 \mathrm{eV}$ are plotted as a function of electron kinetic energy in Figure 5. These energies are chosen as their separation effectively matches that of the principal peaks in the PES, $A-B-C$, and in consequence successive peak positions fall at common electron energies. From this figure one sees consistent $\sim 10 \%$ difference in the PECD in peak areas $A$ and $B$, and similarly a $\sim 5 \%$ difference in the $B$ and $C$ peaks' PECD. Clearly the experimental PECD of each of the assigned vibrational peaks $A, B, C$ displays a relatively small KE dependence and the vibrational modulation appears the more significant factor - at least in the restricted photon energy range $<1 \mathrm{eV}$ examined here.

\section{C: Vibrational Assignments}

Frequencies and normal mode coordinates for the neutral and cation ground states have been calculated by harmonic vibrational analysis using both MP2 and DFT methods. The 3-carene molecule has 72 normal modes, but several of these are deformations extending over the full molecular skeleton mixed with torsional motions of the attached $\mathrm{CH}_{3}$ groups and have calculated frequencies less than $200 \mathrm{~cm}^{-1}$. These are probably poorly described in the harmonic approximation, and strongly coupled. Even in the harmonic model, these modes are strongly mixed on excitation by Duschinsky rotation, and give rise to many combination bands, involving multiple modes and possibly multiple quanta in each participating low frequency mode. In fact, we estimate that across the HOMO band less than $0.6 \%$ of the total excitation intensity arises as simple fundamental or overtone bands, and less than $3.6 \%$ as two-mode combination bands; the bulk of the vibrational excitation occurs by 
combinations of three or more modes. Nevertheless, the visual appearance of the experimental band (peaks $A-B-C-D$ ) is suggestive of a rather simple progression.

Figure 6 shows a $0 \mathrm{~K} \mathrm{FC} \mathrm{simulation} \mathrm{for} \mathrm{the} \mathrm{first} \mathrm{member,} \mathrm{peak} A$, of this apparent progression. The simulation was restricted to fundamentals, overtones and combinations of up to 3 different modes. As well as the true $0-0$ vibrational origin transition, the simulation is dominated by combinations comprising permutations of few quanta excitations of modes $v_{1}-v_{3}$. The maximum in the simulated peak profile in Fig. 6 has been aligned with that of the experimental peak. It is, however, apparent that the peak area maximum is significantly displaced towards higher energy from the true adiabatic transition, and by estimating this displacement from the simulation the adiabatic ionization energy of 3-carene can be obtained as $8.385 \mathrm{eV}$.

As the internal energy available to the cation increases, higher frequency modes appear in these simulations, but always in combinations with the increasing permutations of quanta of modes $v_{1}, v_{2}$, $v_{3}$. Consequently, contributions from additional vibrational modes emerge through a "forest" of the accompanying $v_{1}-v_{3}$ excitations. The significance of these higher frequency modes is then somewhat obscured since the full intensity of the mode is distributed across multiple weaker combination transitions.

To overcome this and present a clearer assignment of vibrational structure in the HOMO ionization, the FC simulations were re-run excluding the low frequency $\left(<200 \mathrm{~cm}^{-1}\right)$ modes. The MP2/6-31G** and B3LYP/cc-pVTZ simulations are very similar and the latter is shown in Figure 7. The convolution function ( $F W H M=120 \mathrm{~cm}^{-1}$ ) applied to the stick spectrum is narrower than experiment in recognition that, as seen in Fig. 6, the true experimental width derives largely from the omitted combinations with low frequency modes. Further, the simulated and experimental peak positions are re-aligned in Fig. 7 to facilitate visual comparison and so ignore the expected strong skew of peak profiles to high energy caused by the missing excitations involving low frequency combinations.

The outermost valence orbital in 3-carene is a $\pi$ orbital at the endocyclic $C=C$ double bond. One may therefore predict that the HOMO ionization would leave the bond weakened and excite the cation $\mathrm{C}=\mathrm{C}$ stretching mode. ${ }^{8}$ Indeed, the simulation (Fig. 7) confirms a progression in the $v_{56} \mathrm{C}=\mathrm{C}$ stretching mode (calculated frequency $1564 \mathrm{~cm}^{-1}$ ), essentially matching the coarse experimental SPES vibrational profile. It can also be seen that single quantum excitation of modes $\mathrm{v}_{43}$, a $\mathrm{C}-\mathrm{H}$ wag around the sixmembered ring plus $\mathrm{C}=\mathrm{C}$ stretch (calculated frequency $1374 \mathrm{~cm}^{-1}$ ) and $v_{61}$, a $\mathrm{C}-\mathrm{H}$ stretch in the peripheral methyls (calculated frequency $2996 \mathrm{~cm}^{-1}$ ) may contribute to the prominent experimentally observed peak areas $A$ to $D$. No less revealing, it can be seen that weaker, single quantum excitations of modes $v_{18}$ (a deformation of both 3-and 6-membered rings, calculated frequency $679 \mathrm{~cm}^{-1}$ ) and $v_{19}$ 
(a six-membered ring breathing motion, calculated frequency $732 \mathrm{~cm}^{-1}$ ) are predicted to contribute to the in-filling of the valleys between the major experimental peaks.

\section{D: Impact of Vibrational Mode on PECD}

A complete, quantitative PECD simulation for a system of such complexity -72 normal modes - is currently not a feasible proposition, even without allowance for the anharmonicity and coupling of low frequency modes, and the extensive range of vibrational combinations identified by the FC simulations, as discussed above. However, some useful insight may be available from modelling the PECD behaviour for various quantum levels of single, isolated normal modes.

This study is performed as previously described. ${ }^{21,22,41,42}$ Briefly, the ground and excited states are written as $\Psi^{\prime \prime}=\varsigma^{\prime \prime}\left(\mathbf{r}_{N} ; Q\right) \chi^{\prime \prime}(Q)$ for the $\mathrm{N}$-electron ground state and $\Psi^{+}=\varsigma^{+}\left(\mathbf{r}_{N-1}, \vec{k} ; Q\right) \chi^{+}(Q)$ for the $\mathrm{N}-1$ electron cation plus continuum electron. Here $\mathbf{r}$ indicates the set of electron coordinates, $Q$, the nuclear coordinates and $\vec{k}$ is the photoelectron momentum. Assuming separability of the electronic and vibrational motion, $\varsigma$ and $\chi$ represent, respectively, the purely electronic and vibrational wavefunctions. This Born-Oppenheimer separability is the principal assumption underlying Franck-Condon approximation.

The electric dipole matrix element for the photoionization can then be written

$$
M_{i, n^{\prime \prime}, f, n^{+}}=\left\langle\chi_{n^{+}}^{+}(Q) \cdot D_{i, f}(Q) \cdot \chi_{n^{\prime \prime}}^{\prime \prime}(Q)\right\rangle_{Q}
$$

where now $D_{i, f}(Q)=\left\langle\varsigma^{+}\left(\mathbf{r}_{N-1}, \vec{k} ; Q\right) \hat{\mu} \varsigma^{\prime \prime}\left(\mathbf{r}_{N} ; Q\right)\right\rangle_{\mathbf{r}}$ is the pure electronic matrix element of the dipole operator, parametrically dependent on the nuclear positions, Q. The Franck-Condon approximation deems, as a secondary assumption, that this dependence is negligible, such that $D_{i, f}(Q)$ is treated as a constant term over $Q$. The integration over $Q$ indicated by the subscript to the right of Eq. 3 then simplifies somewhat, becoming an evaluation of the vibrational overlap integral times the constant $D_{i, f}-$ so fully decoupling the electronic excitation from vibrational motion.

Without making this second assumption we retain the possibility of electron-nuclei coupling through the $Q$ coordinate in the complete photoionization matrix elements $M_{i, n^{\prime \prime}, f, n^{+}}$and this is the approach pursued here. For the current polyatomic problem the photoionization characteristics for a given individual mode are assessed by freezing all other vibrational motions at their equilibrium position. The complex dipole matrix elements are evaluated as averages taken across the 1-dimensional normal mode coordinate of the chosen vibration as given by Eq. 3. ${ }^{21,22,42}$ 
Four cation vibration modes have been selected for examination in this fashion, based on the discussion in the previous section and their prominence in the FC simulation shown in Fig. $7:$ the strongly excited $C=C$ stretch mode $v_{56}$ which as just seen is a major (though non-exclusive) contributor to the progression $A-B-C-D$ in the HOMO PES; a second mode, $v_{43}$, also with some $C=C$ stretch character that appears with a strong $n=1$ excitation; and modes $v_{18}$ and $v_{19}$ whose $n=1$ excitations, either alone or in combinations with $v_{56}$, contribute to fill the valleys between the major peaks. The predicted photoionization cross-sections, branching ratios, and chiral $b_{1}^{(+1)}$ angular distribution parameters for these vibrations are presented in Figure 8, while in Table 1 we list the vibrational overlap integrals (Franck-Condon factors) for these modes, levels $n^{+}=0-4$ that could be obtained in the course of these calculations. In performing these calculations care has been taken, using both visual examination and the Duschinsky projection of neutral vibrational motions on to those of the cation, to accurately identify the corresponding vibration for the ground neutral state. This is necessary as on ionization the frequencies in the ion can change by differing amounts, so that a simple identification of modes in neutral and ion based on ordering by frequency alone can mislead.

The simple, one dimensional FC factors in Table 1 concur with the simulation results appearing in Fig. 7 where only single quantum level excitations of modes $v_{18}, v_{19}$ and $v_{43}$ have predicted intensity that exceed $0.2 \%$ of the total band intensity, but levels $n^{+} \leq 3$ for the stretching mode $v_{56}$ meet this threshold. Full numerical agreement with the one-dimensional FC factors (Table 1 ) is not, however, to be expected since the full simulation evaluates multidimensional FC factors incorporating effects of Duschinsky rotation of the excited state normal modes. . $33,43,44^{-}$

Adopting a qualitative Franck-Condon viewpoint, either set of FC factors implies that for the weakly excited modes $v_{18}, v_{19}$, and $v_{43}$, the displacement of $\chi^{+}$along their respective normal coordinates, relative to the ground neutral state, is small such that the vibrational overlap integrals rapidly diminish with increasing $n^{+}$quantum number. A much more significant displacement would be expected for $v_{56}$. Such expectations are borne out by harmonic wavefunction plots in Figures 9 and 10 that compare the calculated vibrational parameters obtained for mode 18 (as an example) with mode 56 . The integration over $Q$ implicit in Eq. 3 effectively averages the pure electronic photoionization matrix elements, $D(Q)$, weighted by the product term $\chi_{n^{+}}^{+}(Q) \chi_{n^{\prime \prime}}^{\prime \prime}(Q)$ that are shown in the top panels of Figs. $9 \& 10$. While harmonic oscillator wavefunctions for odd and even quantum levels have, respectively, odd and even parity there is a symmetry breaking on forming this product due to displacement of the excited equilibrium position along the normal mode coordinate. Nevertheless, with small displacements this may be slight such that, as here in Fig. $9, \chi_{n^{+}=1}^{+}(Q) \chi_{n^{\prime \prime}=0}^{\prime \prime}(Q)$ retains an approximate anti-symmetry about the origin. In the context of an evaluation of pure FC factors, 
integration over the positive and negative domains of $Q$ will, in such cases, produce a partial cancellation and the FC factors will be small indicating only a weak transition is expected.

Still in the context of the FC approximation, the vibrational level dependent cross-sections can be expected to be given by the total ionization cross-section times the relevant FC factor. At first glance, the ionization cross-sections calculated using Eq. 3, and shown in the top subpanels for each mode in Fig. 8, appear consistent with this. However, the calculated $n^{+}=1: n^{+}=0$ vibrational cross-section ratios reveal weak variations across the energy range plotted in Fig 8. Such variability is contrary to FC predictions since the FC factors determining the branching ratio are themselves ionization energy independent. Instead, we must infer that the FC model assumption of constant amplitude electronic matrix elements, independent of $Q$, does not rigorously hold.

Turning now to a discussion of the calculated vibration specific chiral $b_{1}^{p}$ parameters (Fig. 8), it may first be noted that while all the $n=0$ curves are very similar to the earlier calculation made for fixed equilibrium geometry, considerable variations occur for the vibrationally excited results, especially for modes $v_{18}$ and $v_{43}$. This represents a far more dramatic deviation from the FC principle predicting fully decoupled electronic and vibrational effects than do the branching ratios discussed in the preceding paragraph. This can be rationalized by first noting that while the evaluation of angle integrated cross-sections requires no phase information, photoelectron angular distribution parameters incorporate both amplitude and phase information; this is especially the case for PECD which is particularly sensitive, even more than the conventional anisotropy parameter $\beta$, to the scattering phase contained in the complex dipole matrix elements ${ }^{2}, D(Q)$.

Paralleling and extending the previous discussion, a consideration of the $b_{1}^{\{+1\}}$ parameter results for the "weak FC modes", $v_{18}, v_{19}$ and $v_{43}$, needs to consider the consequences of the integration along $Q$ (Eq. 3) for the phase information. Multiplication of complex electronic matrix elements by an odd parity function contributes a phase reversal across the origin. The consequences have been previously elaborated when, as in Fig. 9 here, the weighting factor $\chi_{n^{+}=1}^{+}(Q) \chi_{n^{\prime \prime}=0}^{\prime \prime}(Q)$ retains an approximate anti-symmetry. ${ }^{21}$ In brief, while a partial cancellation of the amplitudes can be expected, as discussed above, when the phase from the positive and negative domains of integration are added, one with a phase reversal, the resultant phase can swing dramatically, with the observed dramatic consequences for the computed angular distribution parameters.

Comparing the $b_{1}^{\{+1\}}(n=1)$ curves to the fixed equilibrium geometry reference result (Fig.8) one sees a large positive displacement (mode 18), a large negative displacement (mode 43), or an overall much 
weaker response (mode 19). While the similar shape of the vibrational factor $\chi_{n^{+}=1}^{+}(Q) \chi_{n^{\prime \prime}=0}^{\prime \prime}(Q)$ is central to the behaviour of all three of these weakly excited vibrations, the specific outcomes for PECD remain intuitively elusive.

The more strongly excited $v_{56} \mathrm{C}=\mathrm{C}$ stretch mode presents a rather different case. A greater normal mode displacement of the equilibrium position can be anticipated owing to the localised $\pi$ electron HOMO inducing a significant $\mathrm{C}=\mathrm{C}$ geometry change when ionized. The MP2/6-31G** optimized $\mathrm{C}=\mathrm{C}$ bond length increases from $1.343 \AA$ in the neutral to $1.420 \AA$ in the cation. A greater displacement of the cation harmonic functions along the normal coordinate are similarly obvious in Fig. 10, which can be compared with Fig. 9. Consequently, a greater degree of vibrational excitation can be expected upon photoionization (based upon FC insights) and the calculations shown in Fig. 8 do indeed indicate this. The calculations for $b_{1}^{\{+1\}}(n=0,1)$ display small differences, the $n=1$ curve following the fixed equilibrium geometry reference particularly closely, but now the $n=2$ curve shows the significant deviation. Looking at the $\chi^{+} \cdot \chi^{\prime \prime}$ weighting factors in Fig. 10 an explanation is now easily found. For this particular example, when projections of the ground state $\chi^{\prime \prime}(n=0)$ onto the displaced and broader $\chi^{+}$functions are taken the longer range spatial regions of the latter are attenuated, to the extent that with both $n^{+}=1$ and $n^{+}=2$ an internal node is effectively removed. Specifically, the weighting product $\chi_{n^{+}=2}^{+}(Q) \chi_{n^{\prime \prime}=0}^{\prime \prime}(Q)$ for this mode has just two loops with a single central nodal point - the same shape as the $n^{+}=1$ product examined in mode 18 (Fig. 9). Hence, the same arguments as before can now rationalise the more marked variation of $b_{1}^{\{+\}}\left(n^{+}=2\right)$ in this more strongly excited mode.

\section{Conclusions}

The $\mathrm{HOMO}^{-1}$ band of the 3-carene photoelectron spectrum presents very well differentiated peaks that are readily ascribed to vibrational excitation of the cation produced. The measured PECD spectra show a strong correlation with the position of these PES peaks, suggesting a vibrational dependence in the chiral angular distribution of photoelectrons as measured by the PECD technique. In order to clearly demonstrate influence of the vibrational excitation per se, as distinct from the variation of electron energy (that by simple energy balance must accompany increasing vibrational excitation at a given photon excitation energy), experimental data sets can be manipulated for presentation in different ways. First, by plotting the PECD as a function of ionization energy for multiple data sets it is clearly demonstrated that the vibrational peak by peak correlation of the carene PECD persists across the photon energy range studied, largely regardless of the changes in electron kinetic energy 
accompanying each different photon energy. Alternatively, a judicious choice of experimental photon energies allows the PECD correlation with different vibrational structures in the PES to be compared whilst maintaining a fixed electron kinetic energy as part of our attempt to separate out the twin final state influences of photoelectron momentum and cation vibrational excitation.

However, a Franck-Condon simulation reveals that the apparent simplicity of the four prominent, regularly spaced peaks in the HOMO band belies a far greater vibrational complexity. There is a high density of vibrational transitions across the HOMO band, due not only to the high number of 72 normal modes in this molecule but, perhaps even more, to the extensive formation of many combination transitions. Nevertheless, evidence is found for a progression in a cation $\mathrm{C}=\mathrm{C}$ double bond stretch, mode 56, buried in the myriad of combinations with permutations of several quanta of the low frequency deformation modes. This complexity precludes any attempt to perform a quantitative theoretical modelling of our experimental results, but theoretical treatments of four selected vibrational modes is undertaken, using a one-dimensional model in which all but the chosen vibration are frozen.

To first validate the potential used to calculate the photoionization electronic dipole matrix elements, a fully frozen calculation at the molecular equilibrium geometry was shown to produce excellent agreement with PECD measurements made at the adiabatic ionization onset, with presumably minimal vibrational excitation. This calculation was then extended to simulate the vibrational influences. Previous work utilising this approach ${ }^{21,22}$ had led to an expectation that the excitation of single quanta of modes having relatively small displacement of the excited state equilibrium position along the normal mode coordinate would probably be necessary for a large vibrational effect to be evident in the PECD. Such expectations may be subtly modified in light of the present results. First, a vibrational mode with only a slight displacement of its equilibrium position in the cation (hence one that in a Franck-Condon picture would appear as a weakly excited transition) is not a sufficient condition to expect a large vibrational PECD effect. Three modes, $v_{18}, v_{19}$ and $v_{43}$, modelled here can be classified so with small FC factors, but only two show really large changes in their PECD to accompany a single quantum excitation. These two modes also show quite opposite displacement of the $n^{+}=1$ PECD. At root we retain the interpretation that very large changes in scattering phase can be induced by such "weak FC" modes, but the observable outcome is then dominated by interference effects that are not amenable to intuitive prediction and classification.

The fourth mode modelled, the $v_{56} \mathrm{C}=\mathrm{C}$ stretch, has a much greater displacement of the cation's equilibrium position that suggests much larger FC factors, and hence stronger vibrational excitation, would be possible. In a previous study ${ }^{22}$ it perhaps seemed that such "strong FC" modes provided the 
normal, relatively unperturbed PECD. It was therefore unexpected to find that for a two-quantum excitation of this mode a large variation in PECD was predicted. In one sense the mechanism is as before; a large variation in the electron scattering phase facilitated by an approximate anti-symmetry in the projection of the neutral ground vibrational state onto the cation vibration. This property of the vibrational wavefunction projection may be somewhat fortuitous, although the conditions that enable it - a softening of the $\mathrm{C}=\mathrm{C}$ bond in the cation that promotes a longer bond length and a shift to lower frequency - are hardly unique. Hence, it may still be seen that big fluctuations in PECD likely accompany rather weakly excited vibrational levels but a more subtle characterisation of the vibrational modes that facilitate this may be necessary.

There are further conclusions to be drawn from the vibrational behaviours revealed by the simplified one-dimensional calculations in Fig. 8. The vibrational branching ratios are weakly energy dependent, signalling a seemingly weak breakdown of the Franck-Condon approximation. In contrast, the ratio of the vibrational PECD curves, although not explicitly plotted, can be seen to vary far more strongly, constituting a much bigger breakdown of the FC expectations of decoupled electronic and vibrational motions - yet both cross-section and PECD calculations utilise the same electronic matrix elements. This underscores the importance of phase, absent in the cross-sections, in PECD. Moreover, it is now clear from the plots in Fig. 8 that observed differences in PECD from different vibrational levels will also be expected to be electron energy dependent, such that a complete disentanglement of vibration and eKE dependences in PECD cannot be achieved without full supporting theoretical modelling.

Unfortunately, the single, isolated vibrational mode modelling undertaken here is not really sufficient to yield a reliable quantitative prediction for the PECD across the 3-carene HOMO photoelectron band. Even were the modelling to be replicated for all 72 normal modes in 3-carene, the one-dimensional harmonic oscillator treatment omits the consequences of Duschinsky rotation, and the consequent multiple combination transitions, that appear in the multidimensional FC simulation in Fig. 7. Furthermore, recall that this FC simulation itself omits several low frequency modes, multiple permutations of which seem to accompany most of the intensity in the stronger, higher frequency modes. Compounding this issue, the low frequency skeletal deformation modes especially are likely to be anharmonically coupled - all of which can radically and non-intuitively vary the net scattering phase. Nevertheless, the theoretical modelling does yield new insight into how extensively PECD may respond to vibrational motion. In a complementary manner, the alternative experimental plotting modes presented in this article are useful in identifying the scope of vibrational interactions in the photoionization dynamics, for which chiral systems, involving the uniquely phase-sensitive $b_{1}$ observable, are precious templates. 


\section{Acknowledgements}

This research was undertaken as part of the ASPIRE Innovative Training Network, which has received funding from the European Union's Horizon 2020 research and innovation programme under the Marie Sklodowska-Curie Grant Agreement No. 674960. HG acknowledges an ESR fellowship provided by Aspire.

We acknowledge the provision of beamtime by Synchrotron Soleil (beamtime Proposal Nos. 20151368,20171530 ) and we thank the technical staff at Soleil for their support and for the smooth operation of the facility.

We are grateful for access to the University of Nottingham High Performance Computing Facility in support of the computational effort.

Rim Hadidi is thanked for helping with some data acquisition.

Raw data were generated at the Synchrotron Soleil large scale facility. Derived data supporting the findings of this study are available from the corresponding author upon reasonable request. 


\section{References}

$1 \quad$ B. Ritchie, Phys. Rev. A 13, 1411 (1976).

2 I. Powis, in Adv. Chem. Phys., edited by J. C. Light (Wiley, New York, 2008), Vol. 138, pp. 267.

3 L. Nahon, G. A. Garcia, and I. Powis, J. Elec. Spec. Rel. Phen. 204, 322 (2015).

$4 \quad$ M. H. M. Janssen and I. Powis, Phys. Chem. Chem. Phys. 16, 856 (2014).

$5 \quad$ S. Turchini, Journal of Physics: Condensed Matter 29, 503001 (2017).

G. A. Garcia, H. Soldi-Lose, L. Nahon, and I. Powis, J. Phys. Chem. A 114, 847 (2010). L. Nahon, G. A. Garcia, C. J. Harding, E. A. Mikajlo, and I. Powis, J. Chem. Phys. 125, 114309 (2006).

H. Ganjitabar, R. Hadidi, G. Garcia, L. Nahon, and I. Powis, J. Mol. Spec. 353, 11 (2018).

L. Nahon, L. Nag, G. A. Garcia, I. Myrgorodska, U. Meierhenrich, S. Beaulieu, V. Wanie, V. Blanchet, R. Géneaux, and I. Powis, Phys. Chem. Chem. Phys. 18, 12696 (2016).

S. Turchini, D. Catone, G. Contini, N. Zema, S. Irrera, M. Stener, D. Di Tommaso, P. Decleva, and T. Prosperi, ChemPhysChem 10, 1839 (2009).

C. J. Harding and I. Powis, J. Chem. Phys. 125, 234306 (2006).

S. Turchini, D. Catone, N. Zema, G. Contini, T. Prosperi, P. Decleva, M. Stener, F. Rondino, S. Piccirillo, K. C. Prince, and M. Speranza, ChemPhysChem 14, 1723 (2013).

S. Daly, M. Tia, G. A. Garcia, L. Nahon, and I. Powis, Angewandte Chemie International Edition 55, 11054 (2016).

M. Tia, B. Cunha de Miranda, S. Daly, F. Gaie-Levrel, G. A. Garcia, L. Nahon, and I. Powis, J. Phys. Chem. A 118, 2765 (2014). 
S. Leach, M. Schwell, G. A. Garcia, Y. Benilan, N. Fray, M. C. Gazeau, F. Gaie-Levrel, N. Champion, and J. C. Guillemin, J Chem Phys 139, 184304 (2013).

J. C. Poully, J. P. Schermann, N. Nieuwjaer, F. Lecomte, G. Gregoire, C. Desfrancois, G. A. Garcia, L. Nahon, D. Nandi, L. Poisson, and M. Hochlaf, Phys. Chem. Chem. Phys. 12, 3566 (2010).

M. J. Frisch, G. W. Trucks, H. B. Schlegel, G. E. Scuseria, M. A. Robb, J. R. Cheeseman, G. Scalmani, V. Barone, G. A. Petersson, H. Nakatsuji, X. Li, M. Caricato, A. V. Marenich, J. Bloino, B. G. Janesko, R. Gomperts, B. Mennucci, H. P. Hratchian, J. V. Ortiz, A. F. Izmaylov, J. L. Sonnenberg, D. Williams-Young, F. Ding, F. Lipparini, F. Egidi, J. Goings, B. Peng, A. Petrone, T. Henderson, D. Ranasinghe, V. G. Zakrzewski, J. Gao, N. Rega, G. Zheng, W. Liang, M. Hada, M. Ehara, K. Toyota, R. Fukuda, J. Hasegawa, M. Ishida, T. Nakajima, Y. Honda, O. Kitao, H. Nakai, T. Vreven, K. Throssell, J. J. A. Montgomery, J. E. Peralta, F. Ogliaro, M. Bearpark, J. J. Heyd, E. Brothers, K. N. Kudin, V. N. Staroverov, T. A. Keith, R. Kobayashi, J. Normand, K. Raghavachari, A. Rendell, J. C. Burant, S. S. Iyengar, J. Tomasi, M. Cossi, J. M. Millam, M. Klene, C. Adamo, R. Cammi, J. W. Ochterski, R. L. Martin, K. Morokuma, O. Farkas, J. B. Foresman, and D. J. Fox, Gaussian 16 Revision A.03 (Gaussian Inc., Wallingford, CT, 2016).

I. Pugliesi and K. Muller-Dethlefs, J. Phys. Chem. A 110, 4657 (2006).

D. Dill and J. L. Dehmer, J. Chem. Phys. 61, 692 (1974).

J. W. Davenport, Phys. Rev. Lett. 36, 945 (1976).

I. Powis, J. Chem. Phys. 112, 301 (2000).

P. Downie and I. Powis, J. Chem. Phys. 111, 4535 (1999).

M. Stener, D. D. Tommaso, G. Fronzoni, P. Decleva, and I. Powis, J. Chem. Phys. 124, 024326 (2006).

Suppl. Information Ref. [8]

I. Powis, R. C. Menzies, D. M. P. Holland, A. B. Trofimov, A. D. Skitnevskaya, E. V. Gromov, E. Antonsson, M. Patanen, C. Nicolas, and C. Miron, J. Chem. Phys. 149, 074305 (2018).

I. Powis, M. Patanen, E. Antonsson, C. Nicolas, C. Miron, and D. M. P. Holland, Phys. Rev. A 96, 013413 (2017).

H. Kikuchi, M. Kubo, N. Watanabe, and H. Suzuki, J. Chem. Phys. 119, 729 (2003).

F. Santoro, A. Lami, R. Improta, and V. Barone, J. Chem. Phys. 126, 184102 (2007). 
Table1

One-dimensional Franck-Condon factors, $\operatorname{FCF}\left(n^{+}\right)=\left[\int \chi_{n^{+}}^{+}(Q) \chi_{0}^{\prime \prime}(Q) d Q\right]^{2}$ for photoionization from the neutral ground state $n^{\prime \prime}=0$ to level $n^{+}$of the modes $v_{18}, v_{19}, v_{43}, v_{56}$ in the $\mathrm{HOMO}^{-1}$ cation

\begin{tabular}{|l|l|l|l|l|l|l|}
\hline \multirow{2}{*}{$\begin{array}{l}\text { Mibration } \\
\text { Mode }\end{array}$} & \multicolumn{5}{|c|}{$\mathrm{FCF}\left(n^{+}\right)$} & \multirow{2}{*}{$\sum_{n^{+}=0}^{4} \mathrm{FCF}\left(n^{+}\right)$} \\
\cline { 2 - 7 } & $\boldsymbol{n}^{+}=\mathbf{0}$ & $\boldsymbol{n}^{+}=\mathbf{1}$ & $\boldsymbol{n}^{+}=\mathbf{2}$ & $\boldsymbol{n}^{+}=\mathbf{3}$ & $\boldsymbol{n}^{+}=\mathbf{4}$ & \\
\hline 18 & 0.924 & 0.074 & 0.001 & 0.001 & 0.000 & 1.000 \\
\hline 19 & 0.826 & 0.160 & 0.013 & 0.001 & 0.000 & 1.000 \\
\hline 43 & 0.974 & 0.025 & 0.000 & 0.000 & 0.000 & 1.000 \\
\hline 56 & 0.548 & 0.399 & 0.034 & 0.008 & 0.009 & 0.999 \\
\hline
\end{tabular}




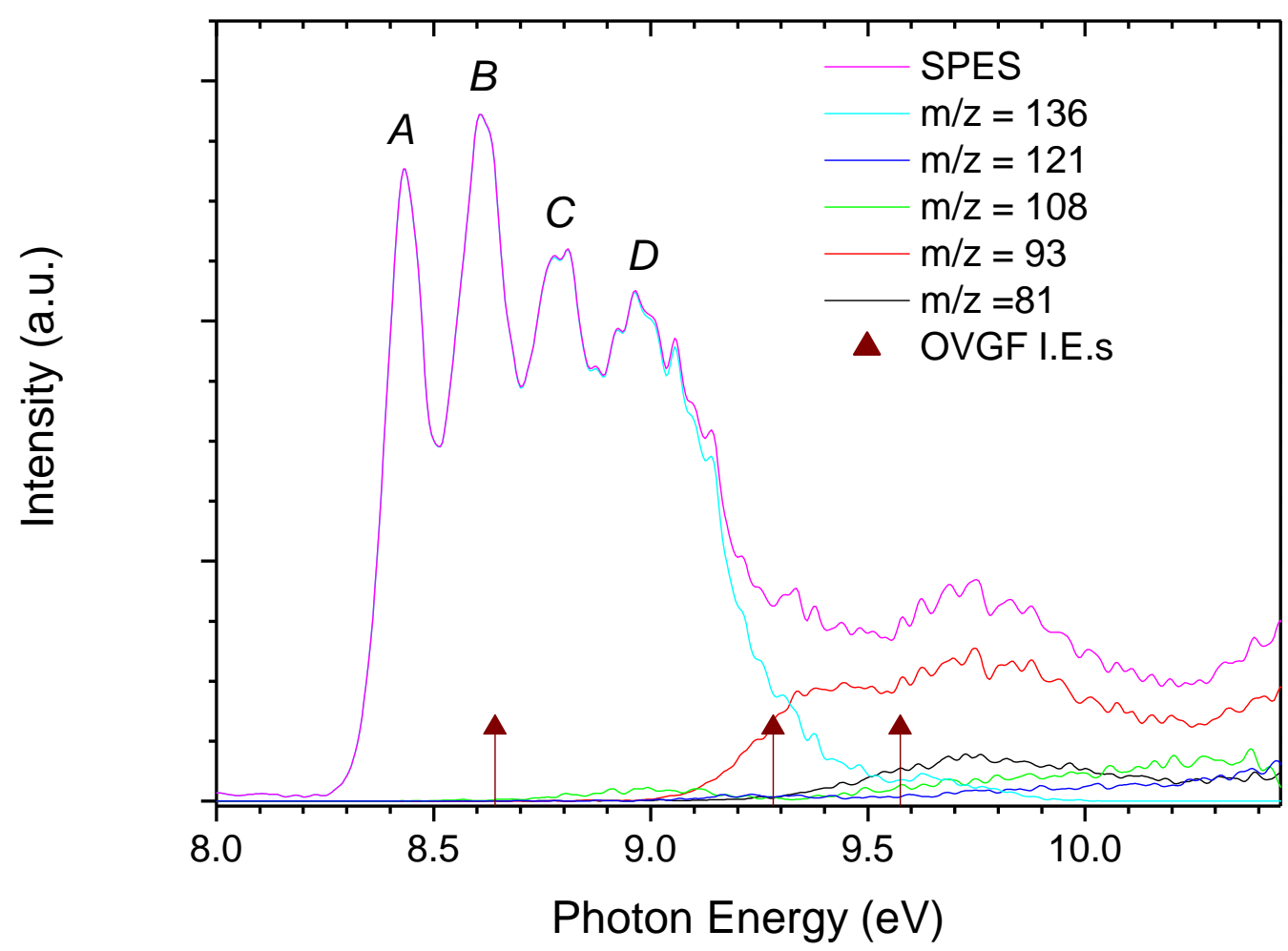

Figure 1.

Total- and Mass-Selected SPES of 3-carene. The principal HOMO peak areas are labelled $A-D$ for ease of subsequent discussion in the text. Calculated vertical ionization energies (OVGF/aug-cc-pVDZ) of the HOMO, HOMO-1, and HOMO-2 orbitals are marked ${ }^{8}$. 


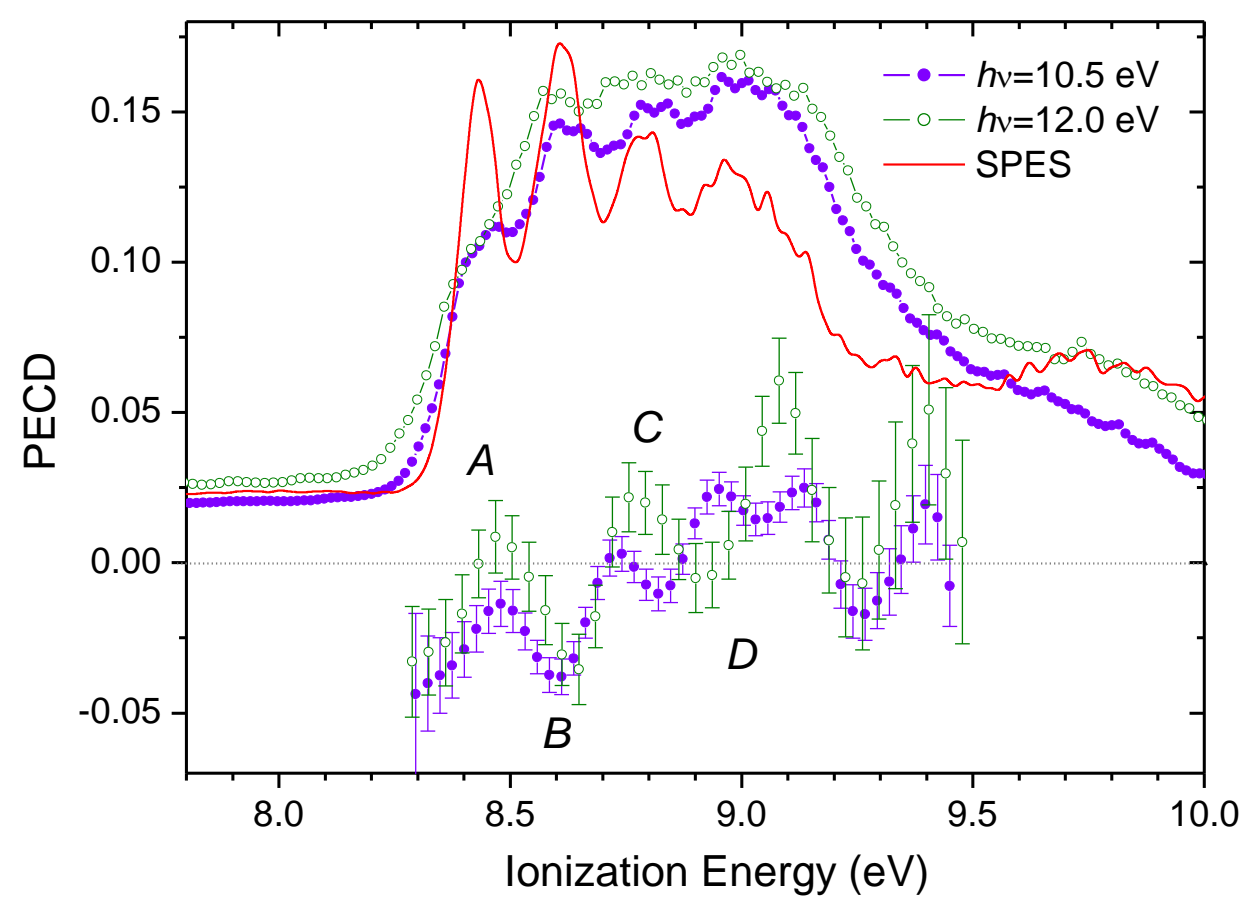

Figure 2.

Examples of the mid-range VMI PECD measurements ( $h v=10.5 \mathrm{eV}, 12.0 \mathrm{eV})$ for $1 \mathrm{~S}-(+)-3$-carene, which here span $\pm 5 \%$. For reference the arbitrarily scaled VMI-PES at these photon energies are plotted above, together with the SPES. Also marked above and below the PECD data are peak areas $A-D$ in the HOMO ionization as identified in the SPES plot (Fig. 1). 


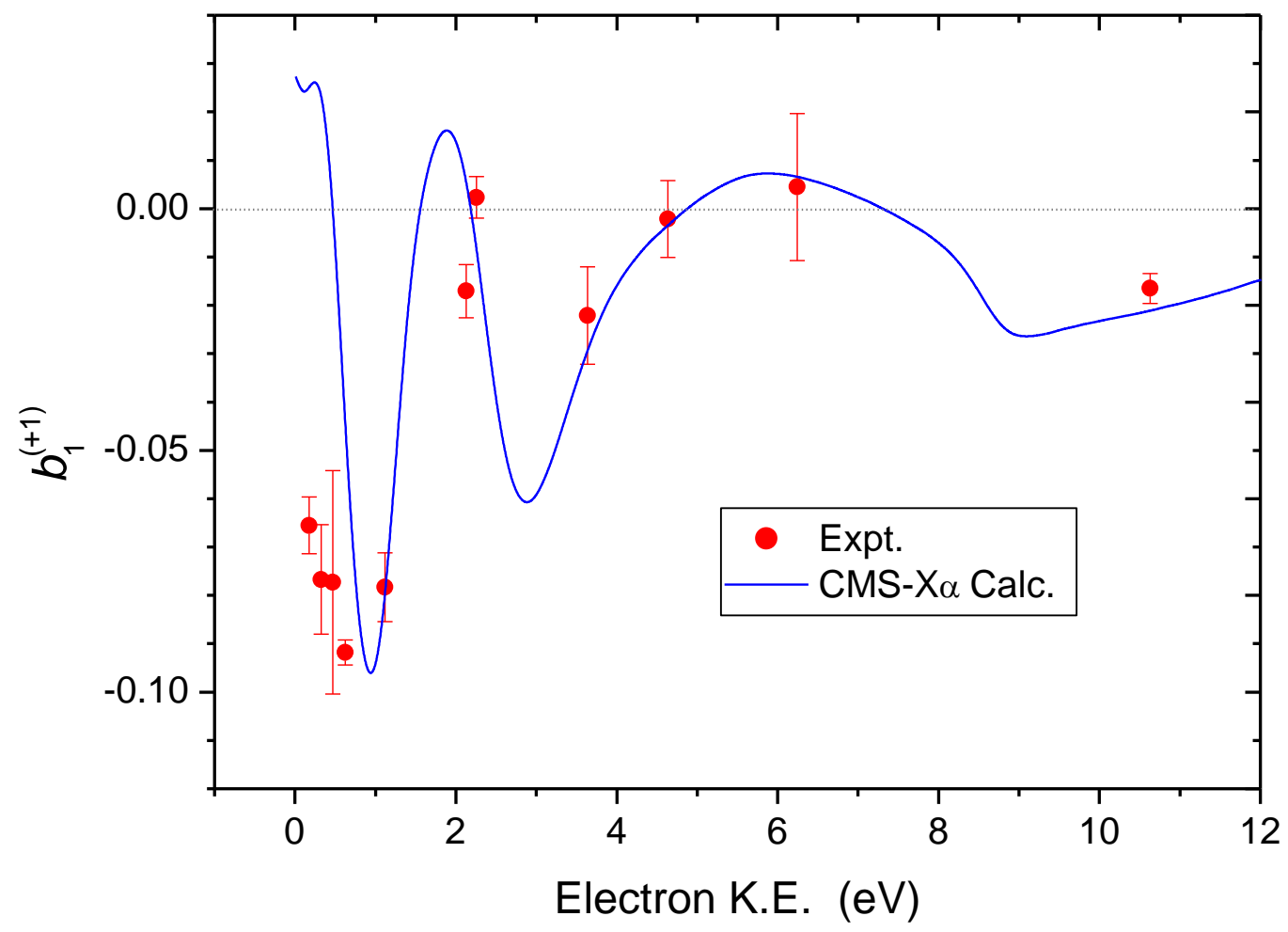

Figure 3.

PECD of (S)-3-carene at the adiabatic (vibrationless) HOMO band origin as a function of electron kinetic energy. Experimental PECD values measured at various photon energies are converted to $b_{1}^{(+1)}$ values for direct comparison with the result of a CMS-Xa calculation for this enantiomer, made for the fixed neutral molecule B3LYP/cc-pVTZ equilibrium geometry. 


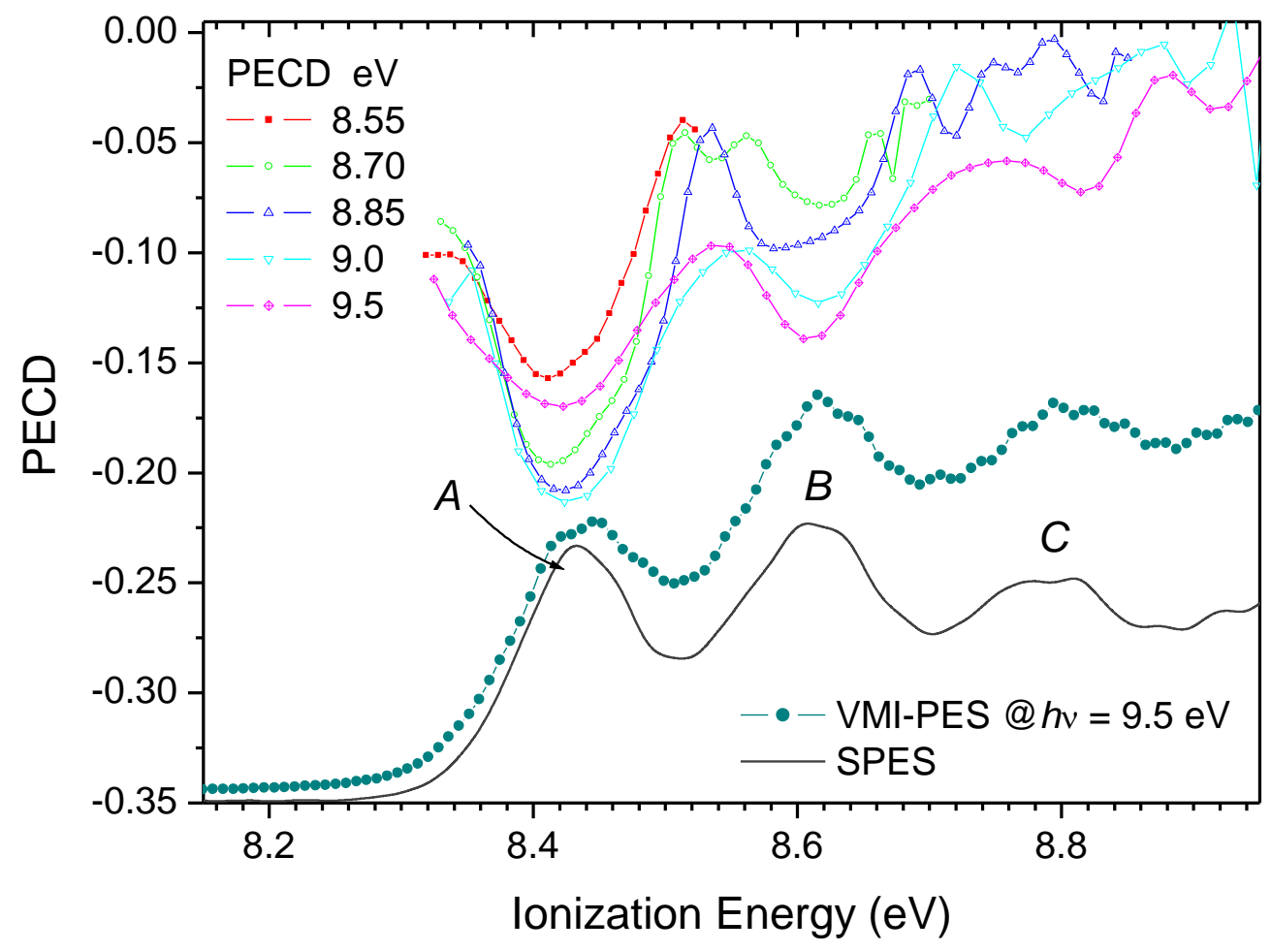

Figure 4.

VMI-PECD measurements of 1S-(+)-3-carene recorded at photon energies $\leq 9.5 \mathrm{eV}$ and recorded in coincidence with parent ion mass ( $\mathrm{m} / \mathrm{z}$ 136), plotted as a function of ionization energy (IE). Error bars have been omitted here for clarity. In the lower half of the figure along the same ionization energy axis are plotted two arbitrarily scaled PES: the VMI-PES recorded at the highest included photon energy, $9.5 \mathrm{eV}$, and the SPES. 


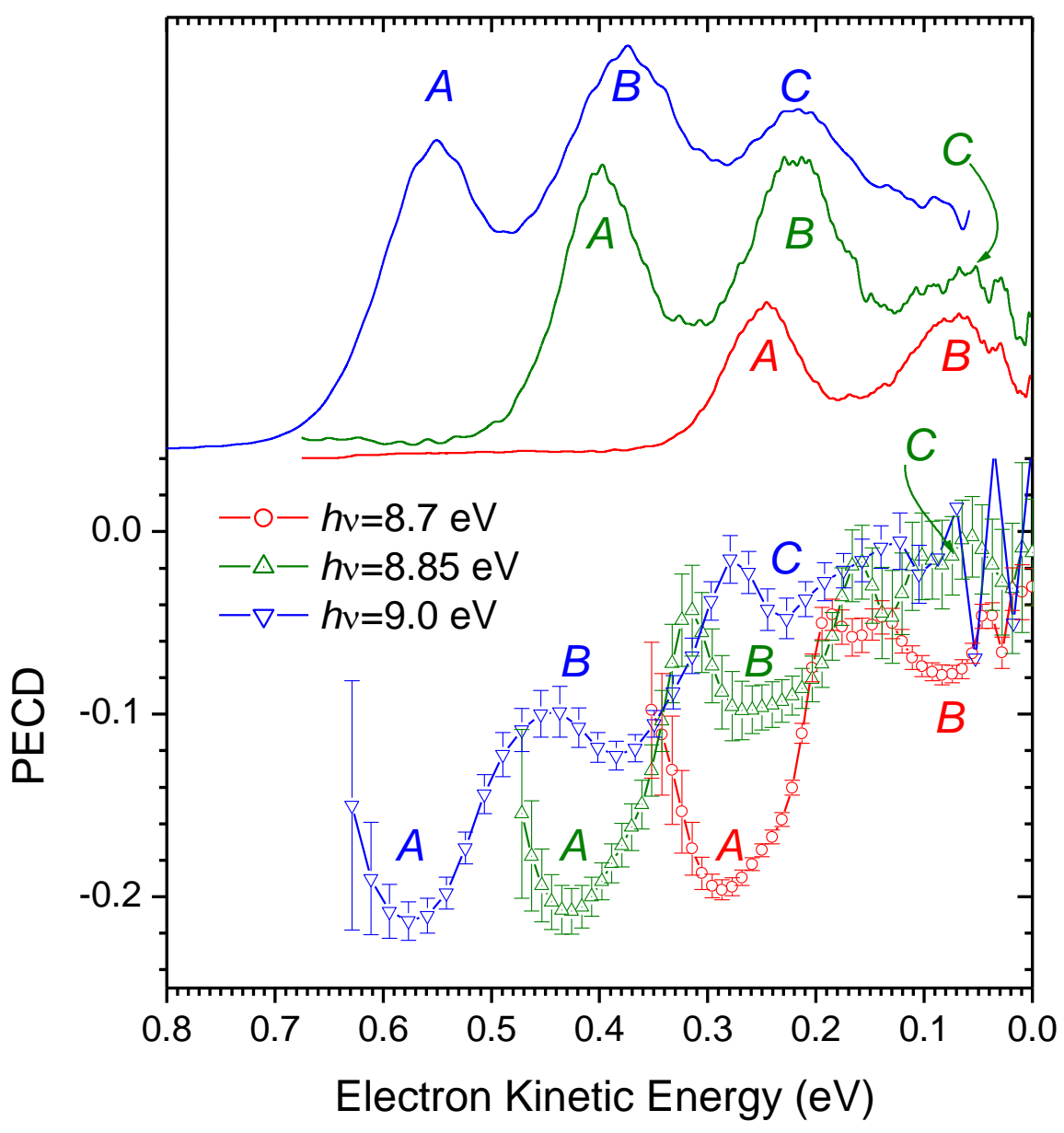

Figure 5

PECD spectra of $1 \mathrm{~S}-(+)-3$-carene recorded in coincidence with the parent mass ions, $\mathrm{m} / \mathrm{z}=136$, at photon energies of $\mathrm{hv}=9.0,8.85,8.7 \mathrm{eV}$. Above these the corresponding VMI-PES are plotted (arbitrarily scaled). Both VMI-PES and VMI-PECD are plotted here as a function of electron kinetic energy. The labels $A, B, C$ that were introduced in Fig. 1 to identify specific peak areas, are applied here (colour-keyed to their respective VMI data) to facilitate discussion. 


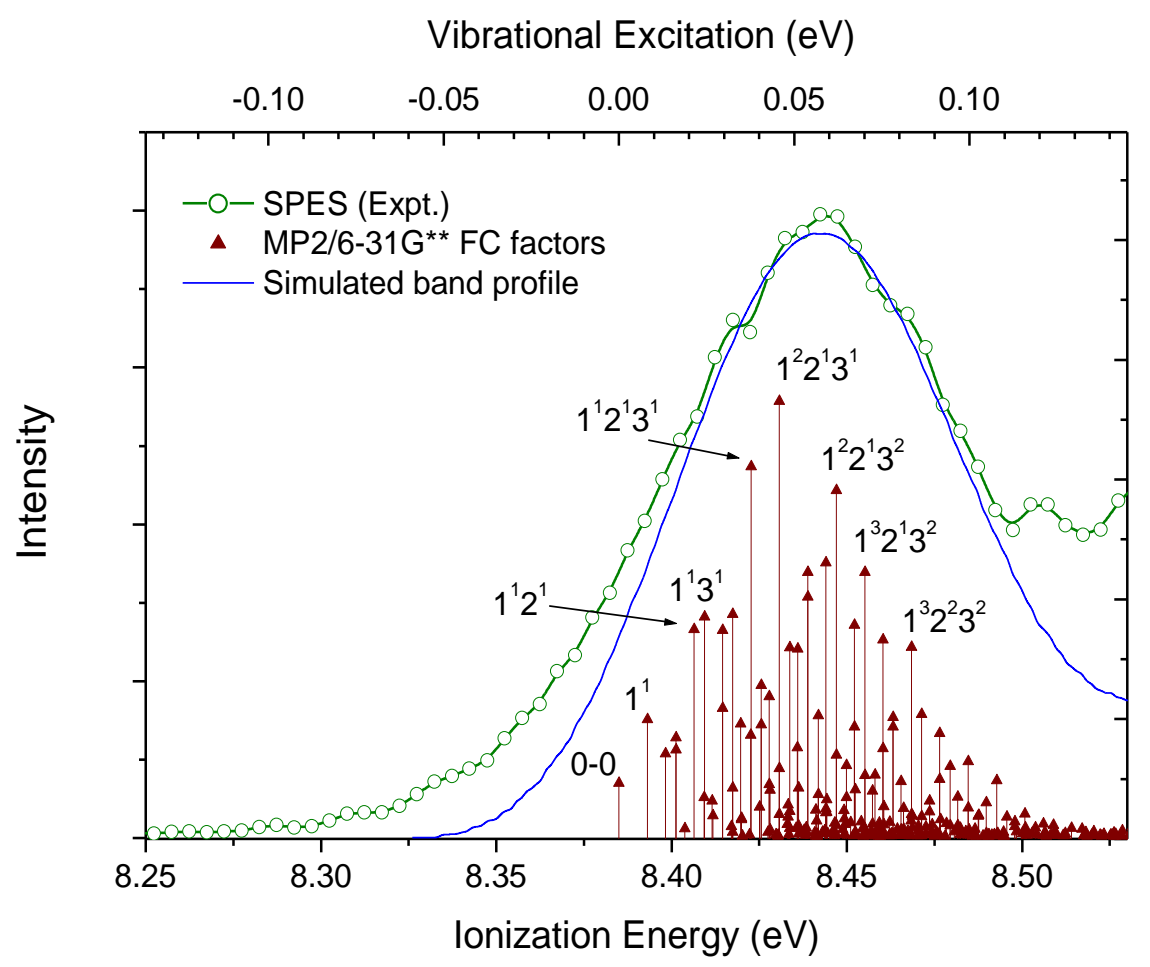

Figure 6

$0 \mathrm{~K}$ Franck-Condon simulation of the first SPES peak, $A$, of 3-carene. Combination bands of up to 3 modes are included in the simulation and example key assignments are shown. 


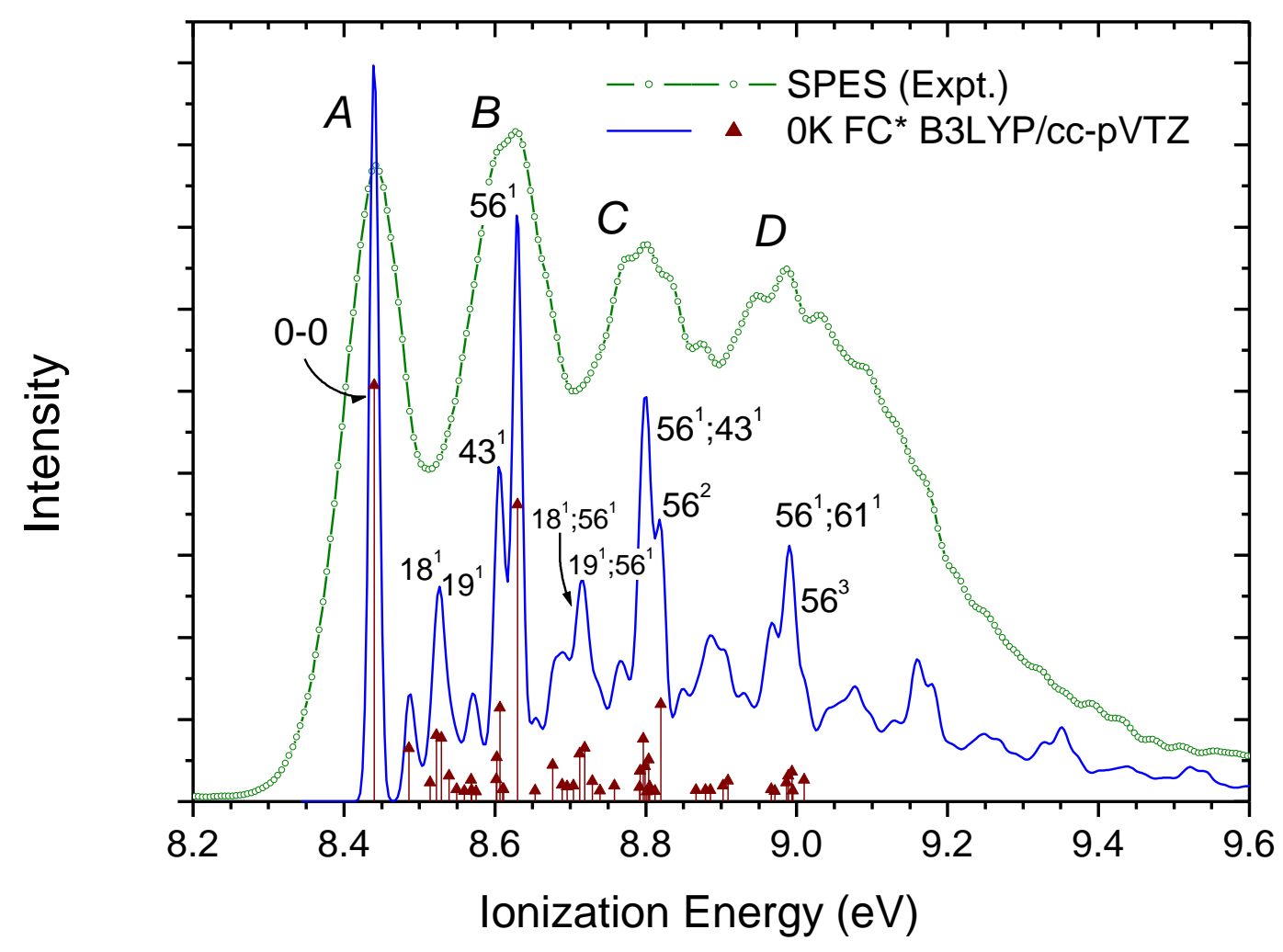

Figure 7

A $0 \mathrm{~K}$ Franck-Condon simulation of 3-carene HOMO PES, excluding modes $<200 \mathrm{~cm}^{-1}$ compared with the experimentally measured SPES. Only the 50 most intense transitions are shown in the calculated stick spectrum, and principal contributions to the convolved peaks are labelled. 

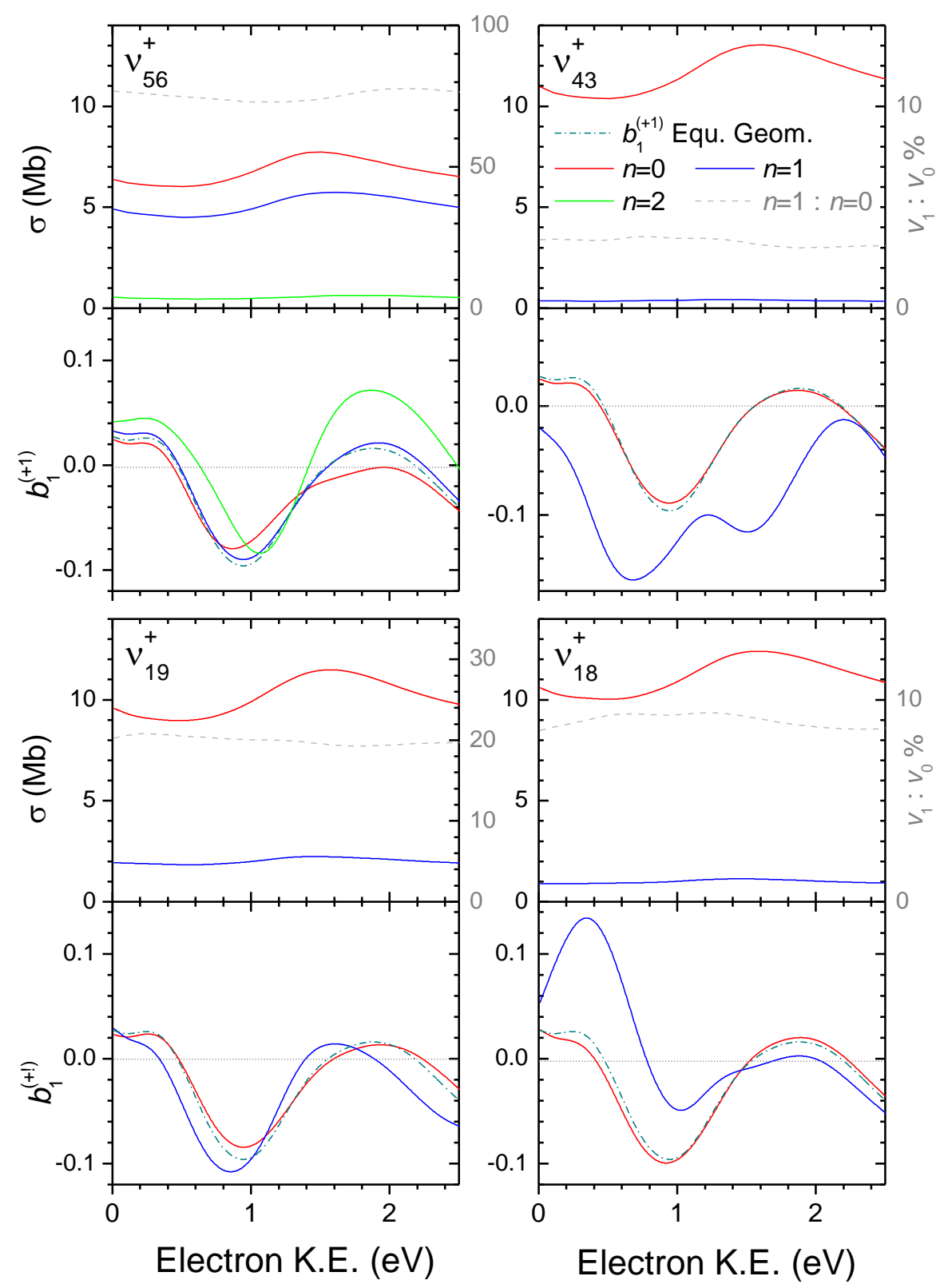

Figure 8

Vibrational mode specific photoionization cross sections and chiral angular distribution parameters $b_{1}^{(+1)}$ calculated for S-(+)-3-carene using complex dipole matrix elements that have been averaged across the normal mode vibrational coordinate. Results are shown for four selected vibrational modes of the cation: $v^{+}=18,19,43,56$. The upper sub-panels showing cross-sections (left axes) also include the percentage ratio of the cross-sections for $n=0,1$ (right axes). For convenience the lower sub-panels showing $b_{1}^{(+1)}$ all include the fixed equilibrium geometry calculation of $b_{1}^{(+1)}$ that appears in Fig. 3. A key for all sub-plots is provided top right. 

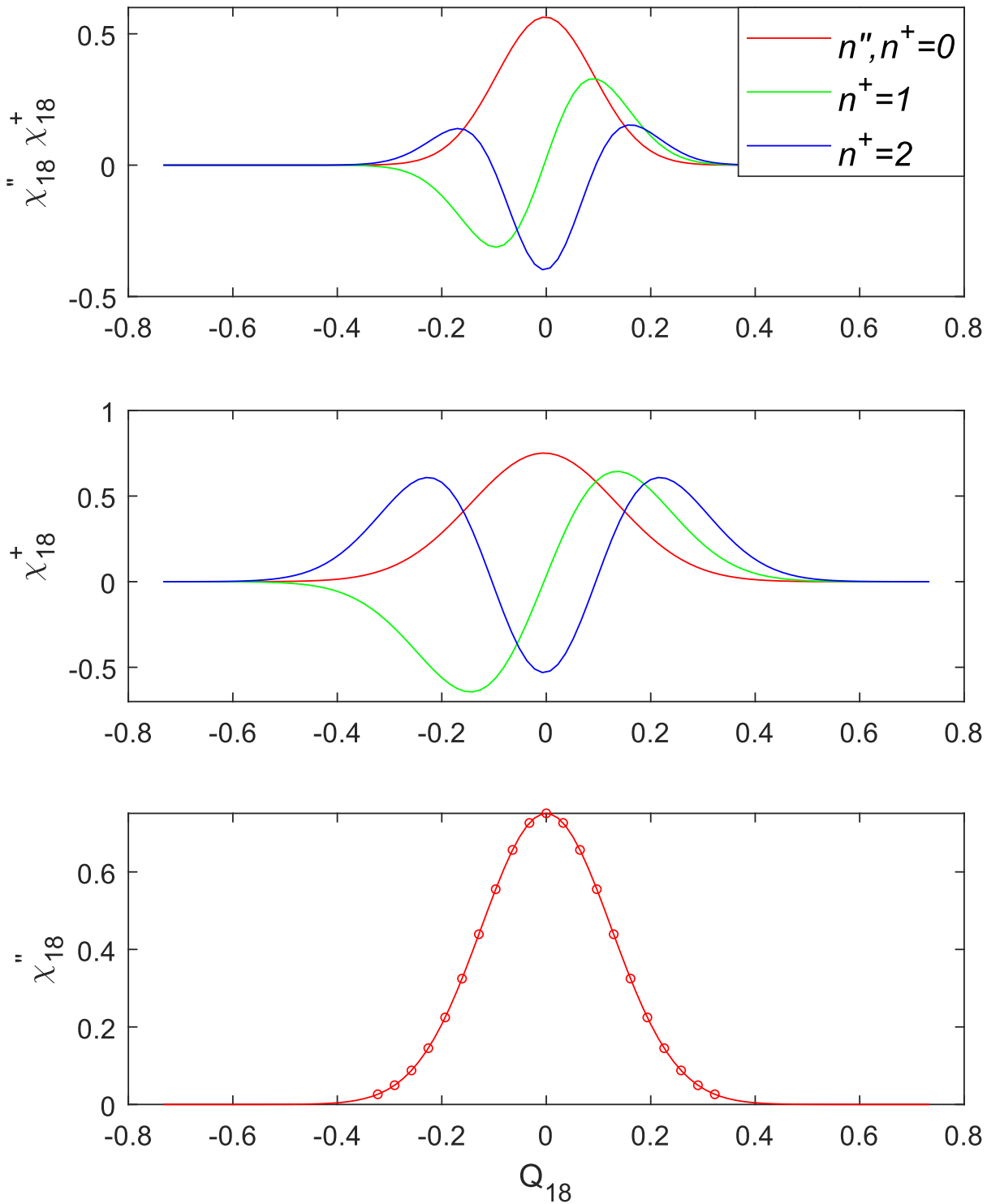

Figure 9

Harmonic vibrational wavefunctions calculated for 3-carene mode 18. Bottom panel shows $\chi^{\prime \prime}\left(n^{\prime \prime}=0\right)$ for the neutral ground state, the middle panel shows the displaced $\chi^{+}\left(n^{+}=0,1,2\right)$ for the $X$ state cation, and the top panel the corresponding product functions, $\chi^{\prime \prime} \chi^{+}$. In the lower panel the points along the normal coordinate, $Q$, at which the matrix elements are evaluated for use in numerical integration/averaging (Eq. 3) are also marked. 

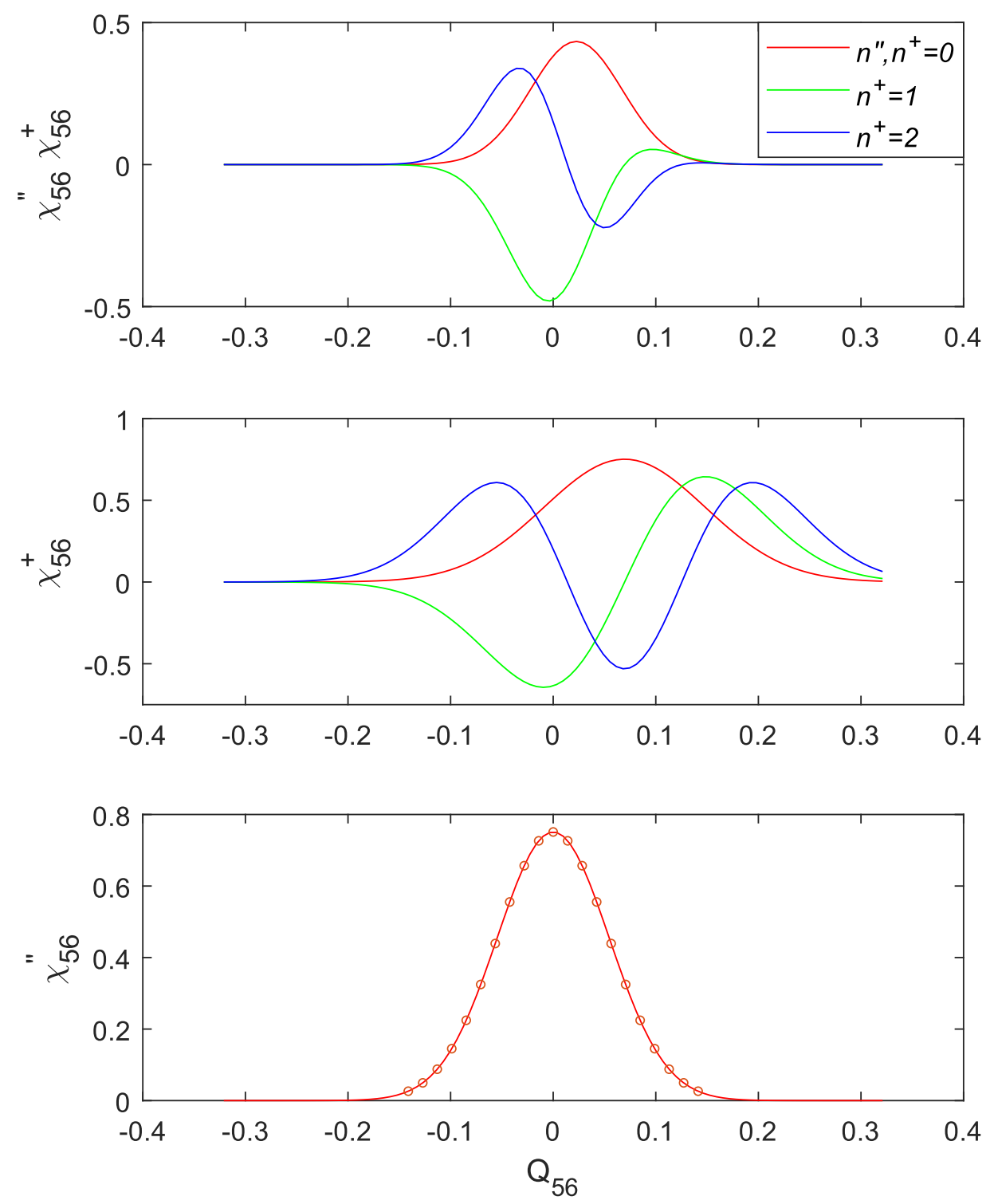

Figure 10

As Fig. 9, but showing results for 3-carene vibrational mode 56. 